\title{
Design of an impact loading machine to determine constitutivelaws at medium or high strain rate.
}

\author{
C. Froustey ${ }^{1}$, S. Denneulin ${ }^{2}$ and P. Viot $^{2}$ \\ ${ }^{1}$ Université Bordeaux 1, 2Arts et Metiers ParisTech, Lamefip Esplanade des Arts et Métiers, F 33405 \\ Talence Cedex (France)
}

The determination of experimental constitutive behaviors at medium or high strain rate is always an important goal. This paper presents a device that has been designed for tensile loading (up to 10s) and some results obtained on different materials: metallic materials and composite materials.

\section{Experimental set-up}

A very widely used technique for straining materials at high rates of strain is based on the so-called Split Hopkinson Pressure Bar (SHPB) [1]. The classical SHPB implies a specific range for specimen dimensions: the specimen must be short enough to minimize the transitory stage leading to quasistatic equilibrium and satisfy a sufficient number of reflections, within the gauge length [2]. Unfortunately, with the SHPB technique, neither the velocity nor the force is imposed. This inconvenience has resulted in apparatus like the flywheel for which the tensile loading is obtained by applying a displacement at a constant velocity [3].

\section{The flywheel apparatus}

Derived from the Charpy pendulum principle, the essential elements of the device are a wheel and a pendulum system. The wheel is large (1 meter diameter), with a mass of $620 \mathrm{~kg}$ and it is equipped with a hammer on its circumference. For storing energy, the wheel turns freely until it reaches the selected speed, then the pendulum system is lowered. The specimen linked with the pendulum system has an anvil fixed at one end. The hammer comes to impinge the anvil and the specimen is then strained. Fig.1 llustrates the test procedure.

With a velocity range from 1 to $40 \mathrm{~m} \mathrm{~s}$, the energy available upon impact (530 J to $200 \mathrm{~kJ}$ ) is far greater than the work needed to break a specimen. Thus, the loading velocity remains almost constant during the test. Moreover, the relative dimensions of the disk diameter and specimen length implies that the loading can be considered as a pure tensile loading.

The force is measured by a long type transducer bar. It consists of strain gauges set on the bar of the pendulum. This force transducer offers a study range linked with the diameter and the material of the installed bar. For example, with a brass bar and a diameter of $20 \mathrm{~mm}$, the capabilities are from 1 $\mathrm{kN}$ to $50 \mathrm{kN}$.

This is an Open Access article distributed under the terms of the Creative Commons Attribution-Noncommercial License 3.0, which permits unrestricted use, distribution, and reproduction in any noncommercial medium, provided the original work is properly cited. 


\subsection{Multi-CCD camera system and displacement measurements:}

A multi-CCD camera system, which is synchronized with the force sensor signal, is dedicated to the displacement measurement. It gives both qualitative and quantitative information on the physical strain mechanism leading to the breaking of the specimen. This is a multi-component system, composed of $8 \mathrm{CCD}$ technology cameras, a sequencer which is the control element and a flash lamp.

To obtain the longitudinal displacement values, specific instrumentation and methodology were defined. The specimens are instrumented using small round markers and after an impact tensile test, a group of three values is obtained: ordinate point, time, and longitudinal strain. To determine the transverse displacement, the current diameter or current height of the specimen is monitored. We can first deduce the current cross-section and then, using the force signal, determine the normal stress.

As a consequence, from the measurement of the force, of the longitudinal and the transverse displacements, it is possible to determine the mechanical behavior of the material.

\section{Experimental results}

Different type of materials and different specimen geometries can be investigated on this tensile impact loading device.

We propose to present some results obtained on metallic and composite materials. The metallic materials are aluminium alloys (2000 and 5000 series) and a steel with high mechanical characteristics. For the composite materials, the investigations concern kevlar 129 epoxy matrix and carbone with polyamide thermoplasitic-matrix. Fig. 2 illustrates the mechanical tensile behavior of the 5000 serie (Al-Mg alloy).

\section{References}

[1] J.E. Field and S.M. Walley and W.G. Proud and H.T. Goldrein and C.R. Siviour. Review of experimental techniques for high rate deformation and shock studies. International Journal of Impact Engineering. 2004, vol30, pp725-775

[2] J.L. Lataillade. Structural Components. Mechanical Tests and Behavioral Laws. Chapter 9: Dynamic Tests. Wiley-ISTE, 2008, pp225-291

[3] C. Froustey and M. Lambert and J.L. Charles and J.L. Lataillade. Design of an impact loading machine based on a flywheel device: application to the fatigue resistance of the high rate prestraining sensitivity of aluminium alloys. Experimental Mechanics, 2007, vol47(6), pp709-721

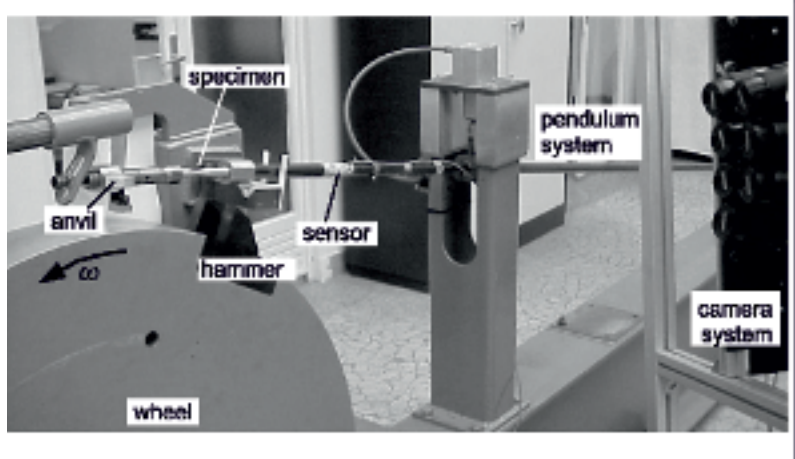

Figure 1

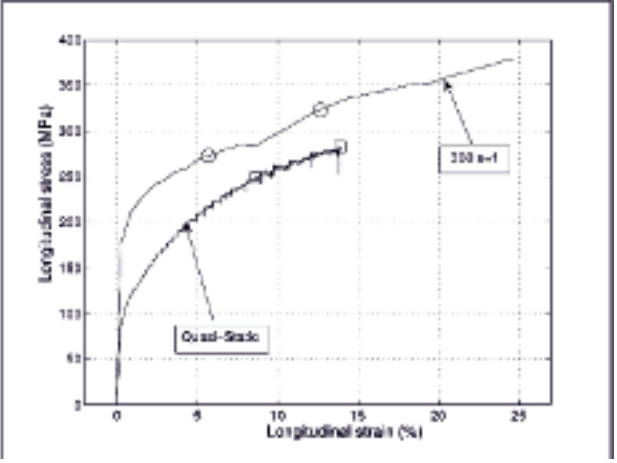

Figure 2 